\title{
LIDAR Echo Emulator
}

10 October 2018

Pawel Adamiec, Alvaro Machon, Monica Rodriguez Cortina, Alejandro Lopez-Moya, Enrique Cordero, Juan Barbero 


\section{CONTENTS}

- Introduction

- Requirements

- Design and manufacturing

- Results

- Conclusions 
- Lidar is a powerful tool in a wide field of applications like: remote sensing study of the atmosphere, temperature profiling, wind velocity probing, 3D imaging, mapping, etc.

- The most critical parts are the photonic components: emitters and receivers.

- Promising candidate is a $\mathrm{HgCdTe}$ avalanche photodiode (APD) which can be used in application demanding detection of low number of photons within the observation time.

- Laboratory evaluation of the receiver with Emulated LIDAR echoes 
- Lidar is a powerful tool in a wide field of applications like: remote sensing study of the atmosphere, temperature profiling, wind velocity probing, 3D imaging, mapping, etc

- The most critical parts are the photonic components: emitters and receivers.

- Promising candidate is a HgCdTe avalanche photodiode (APD) which can be used in application demanding detection of low number of photons within the observation time.

- Laboratory evaluation of the receiver with Emulated LIDAR echoes
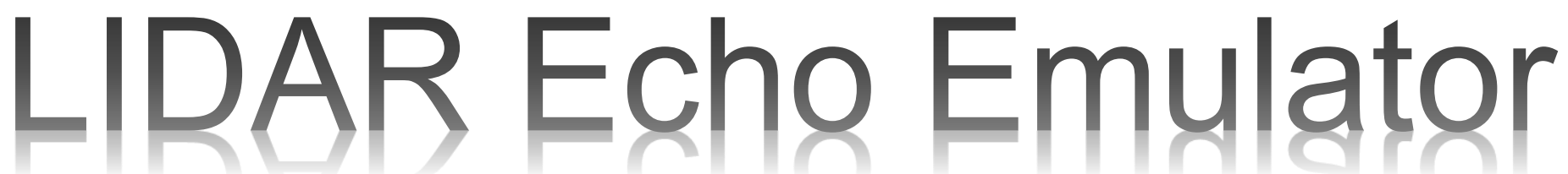


\section{LIDAR Echo Emulator REQUIREMENTS}

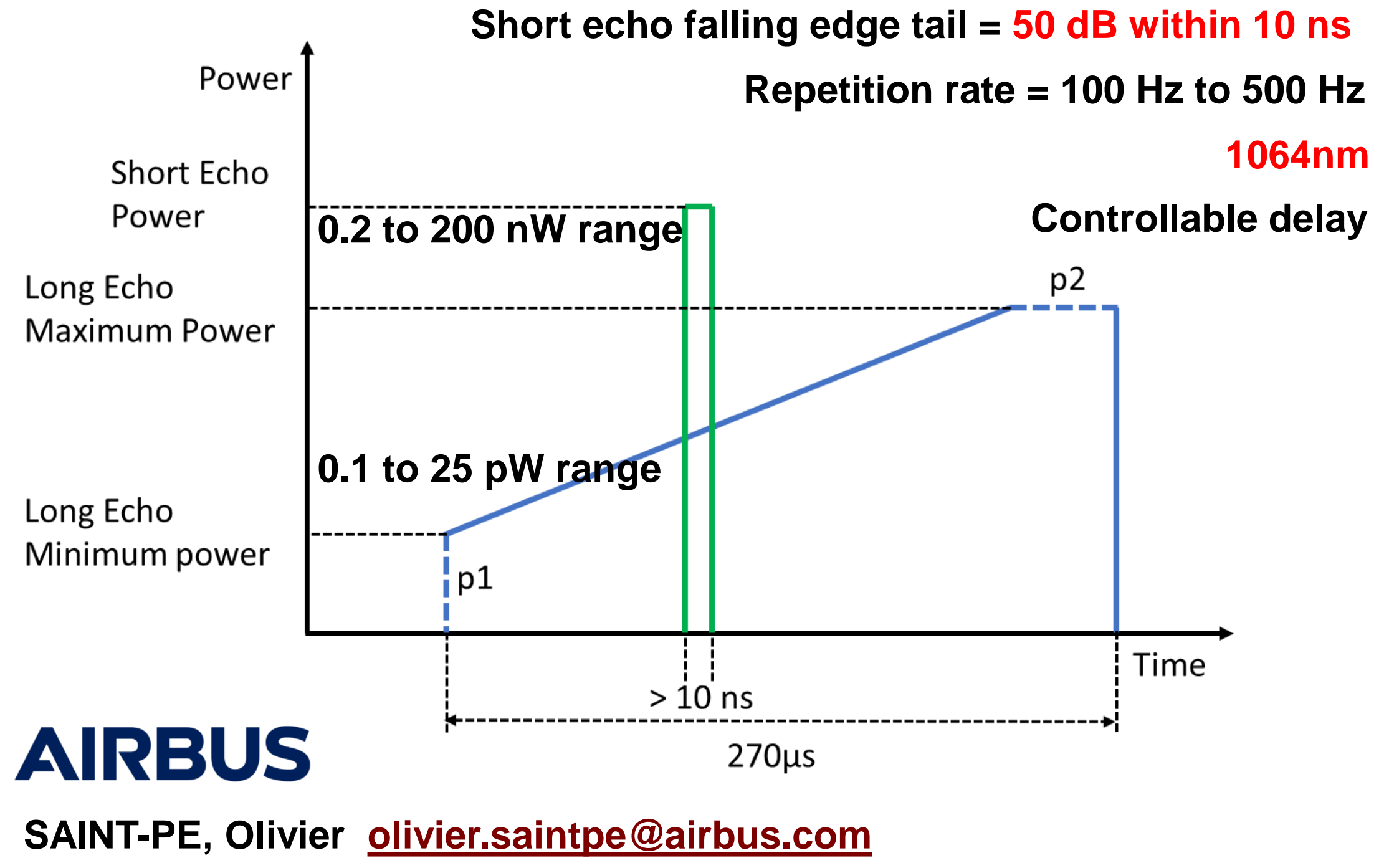




\section{LIDAR Echo Emulator Basic Design}

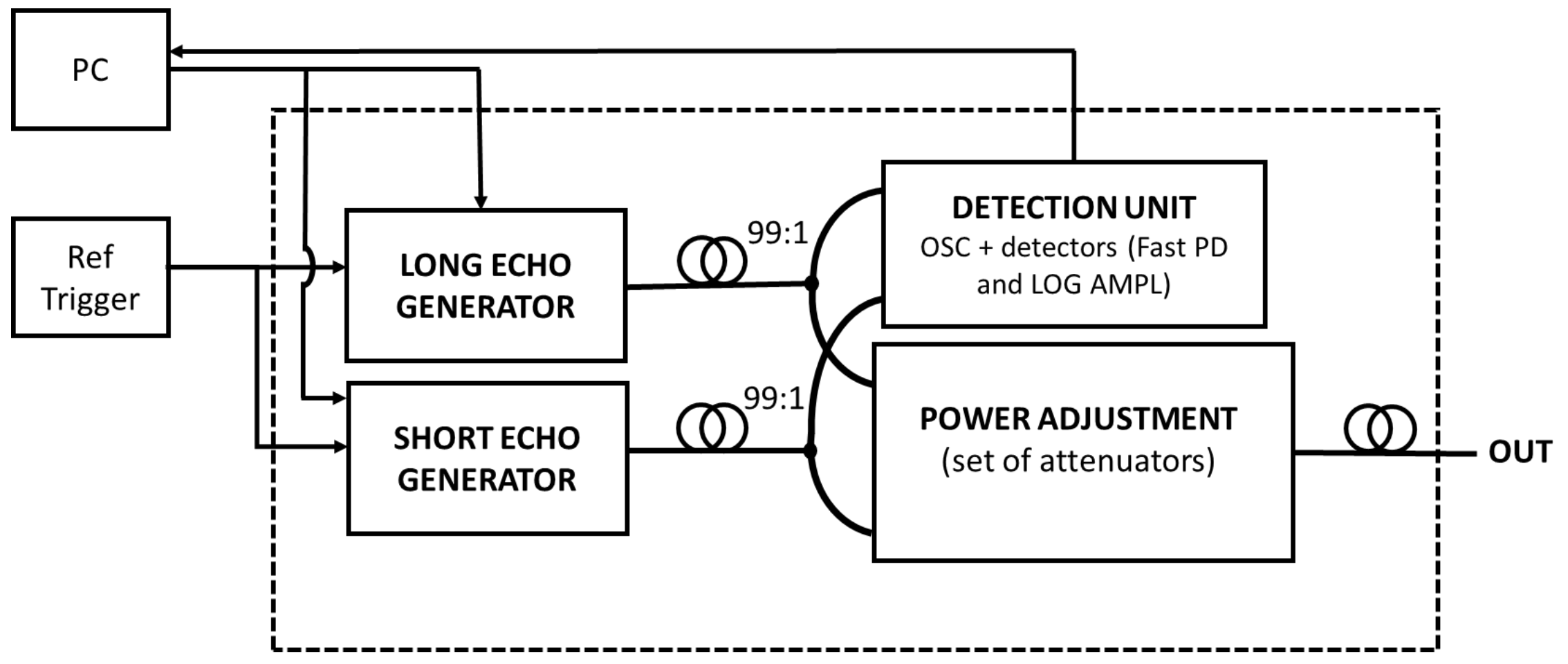




\section{LIDAR Echo Emulator Basic Options}

EMITTER options analyzed

1. Directly modulated Laser

- Arbitrary Wavefront Generator + DFB LASER

2. Diode Pumped Solid-State (DPSS) laser

Non-Linear Crystal

- Pulse laser

3. Optical Modulation

- LiNbO3 modulators

- AOM

- OES

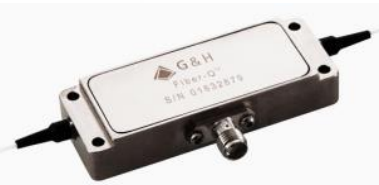

DETECTOR

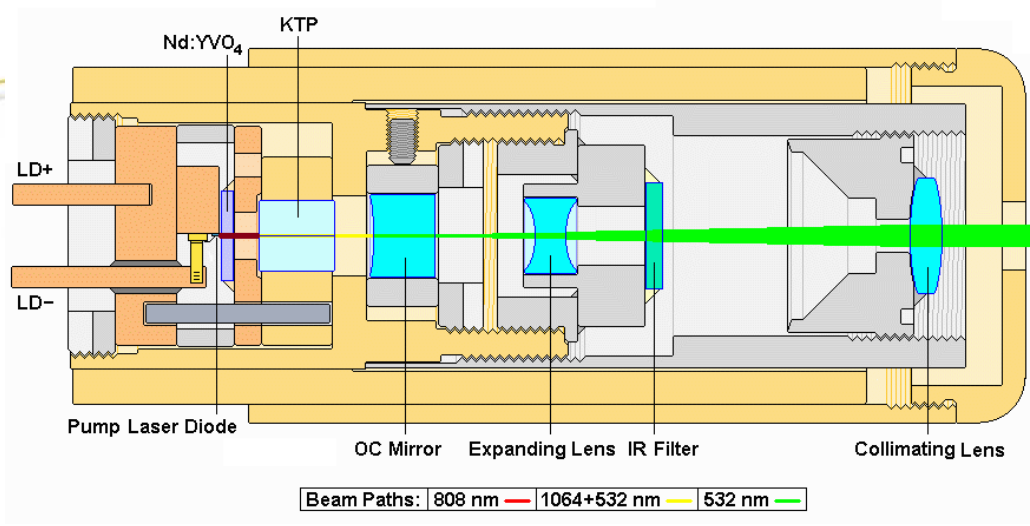

1. PHOTODIODES

- Scope integrated in the setup

- Logarithmic Amplifiers for 50dB range

SOFTWARE

1. LABVIEW 


\section{LIDAR Echo Emulator Basic Options}

\section{Cascade Switches}

OES technology for $1550 \mathrm{~nm}$ from EpiPhotonics (EPS0102).
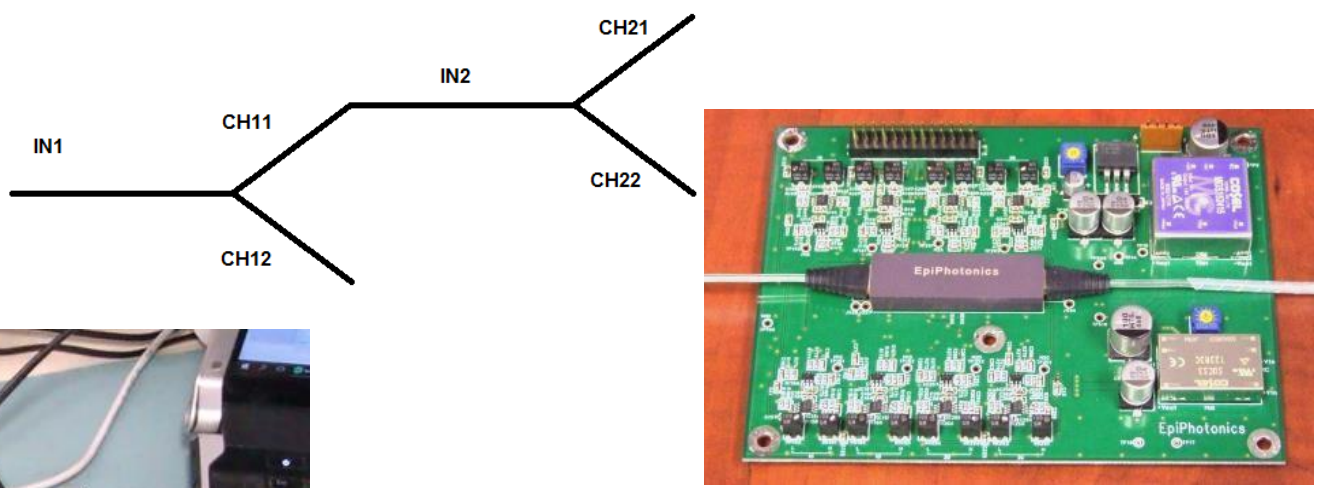

25 ns on-off time $45 \mathrm{~dB}$ dynamic range
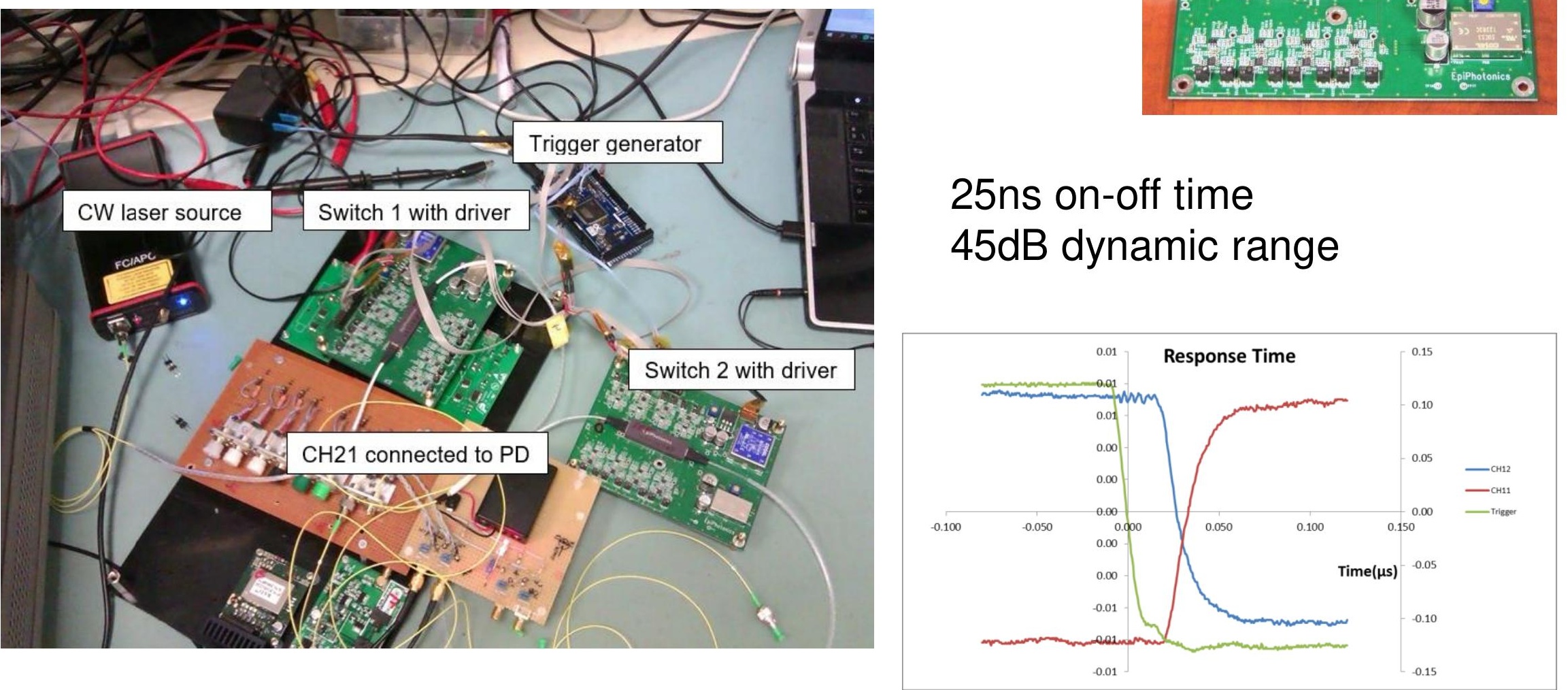


\section{AWG + Laser driver}

\section{MODULAR EQUIPMENT}

- AWG Tektronics AFG320

- THORLABS LASER driver

- Scope Tek

PicoLAS

- AWG PLCS-40

- LASER driver BFS-VRM 03 HP
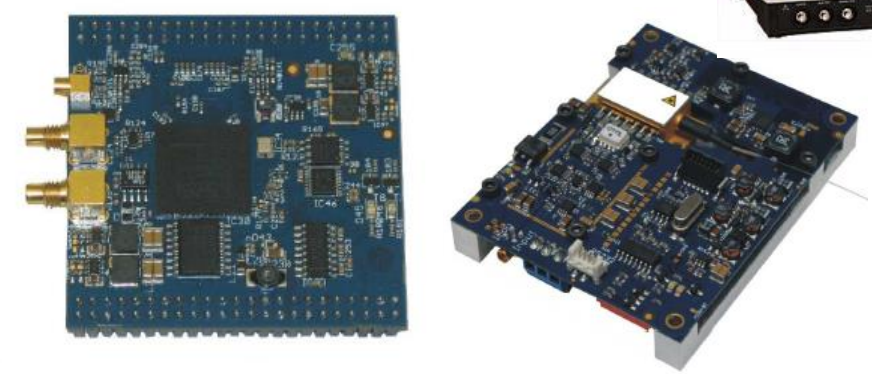

OPTICAL PULSE MACHINES OPM-LD-pS PULSE SHAPING

1. Multiple functions integrated in one unit:

- Arbitrary waveform generator

- High bandwidth linear current driver

- Efficient TEC controller

- Laser diode

2. OPM's AnypulseTM technology

3. Flexible pulse shaping with $\mathbf{1}$ ns resolution

4. Laser current up to $2 \mathrm{~A}$ with 11 bit resolution

5. Triggering modes: Internal, external, loops

6. Serial interface to PC or to customer's system

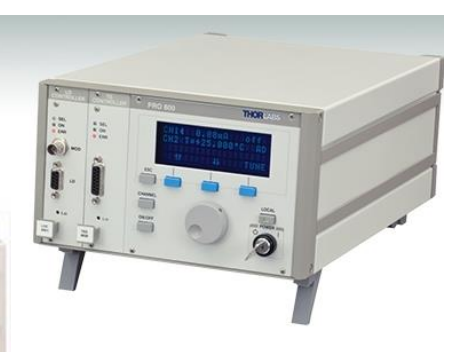

\section{NOTICE AWLDD}

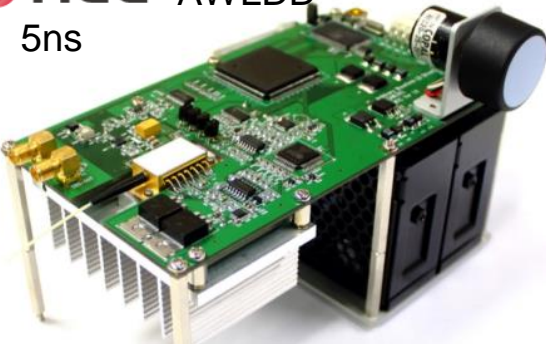

HIGHLAND T160 picosecond-nanosecond diode laser driver
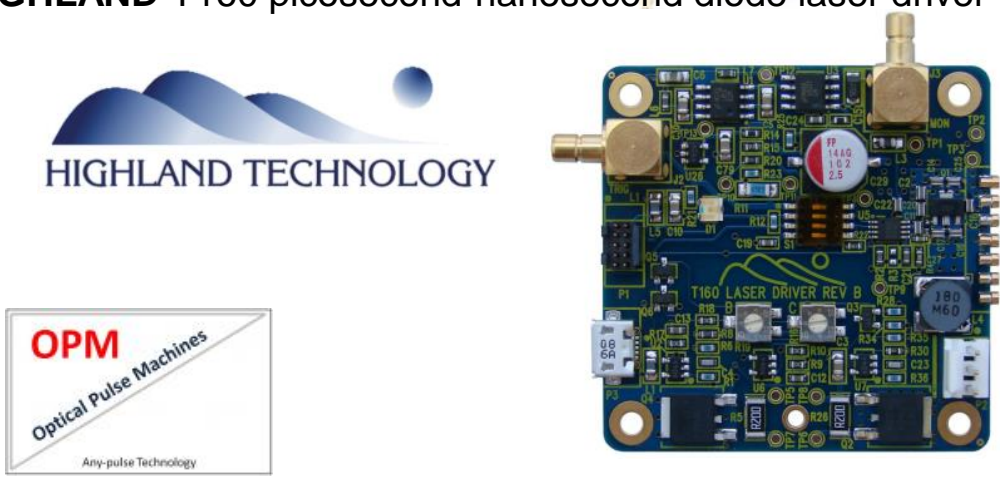

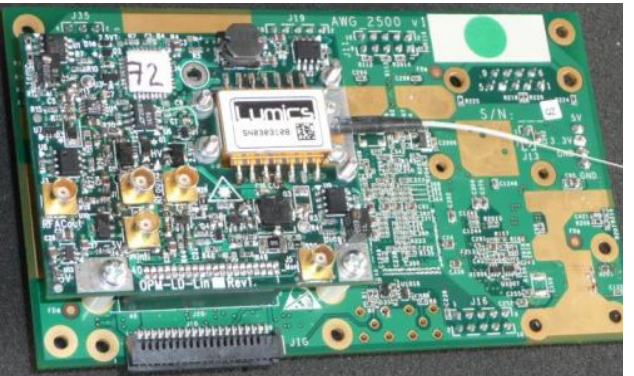




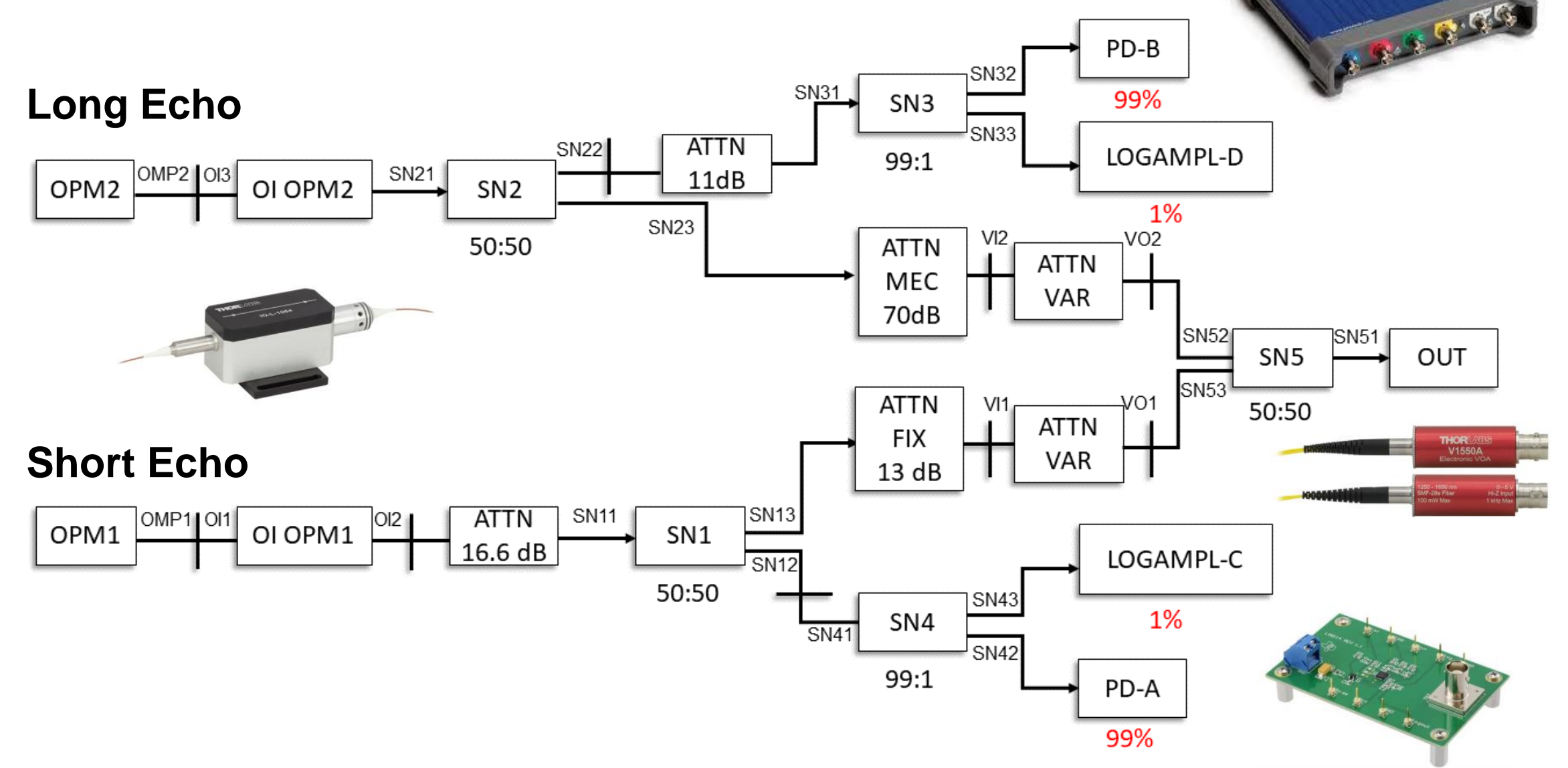




\section{LIDAR Echo Emulator Basic Design}
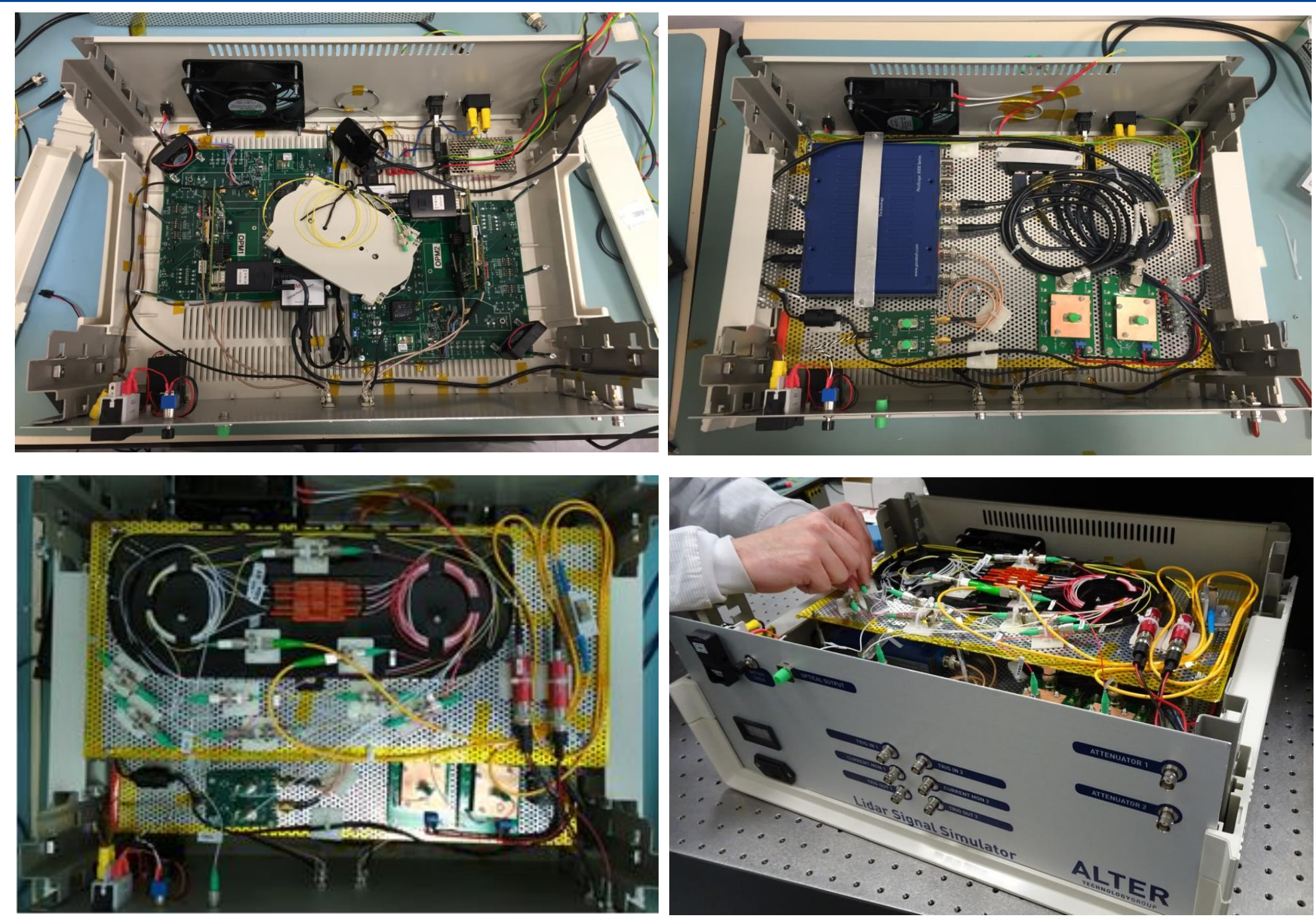


\section{LIDAR Echo Emulator Software}

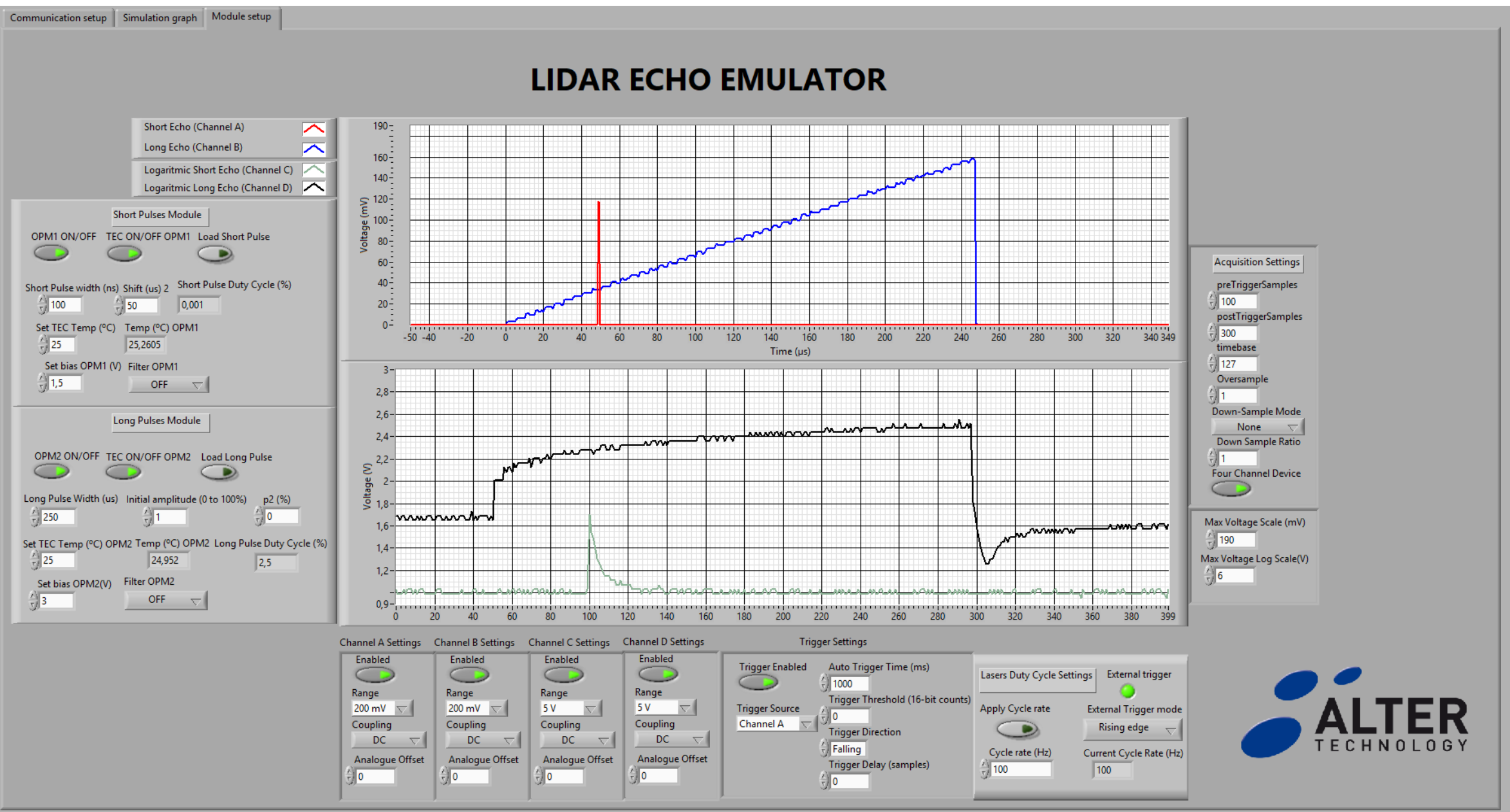




\section{LIDAR Echo Pulse examples}

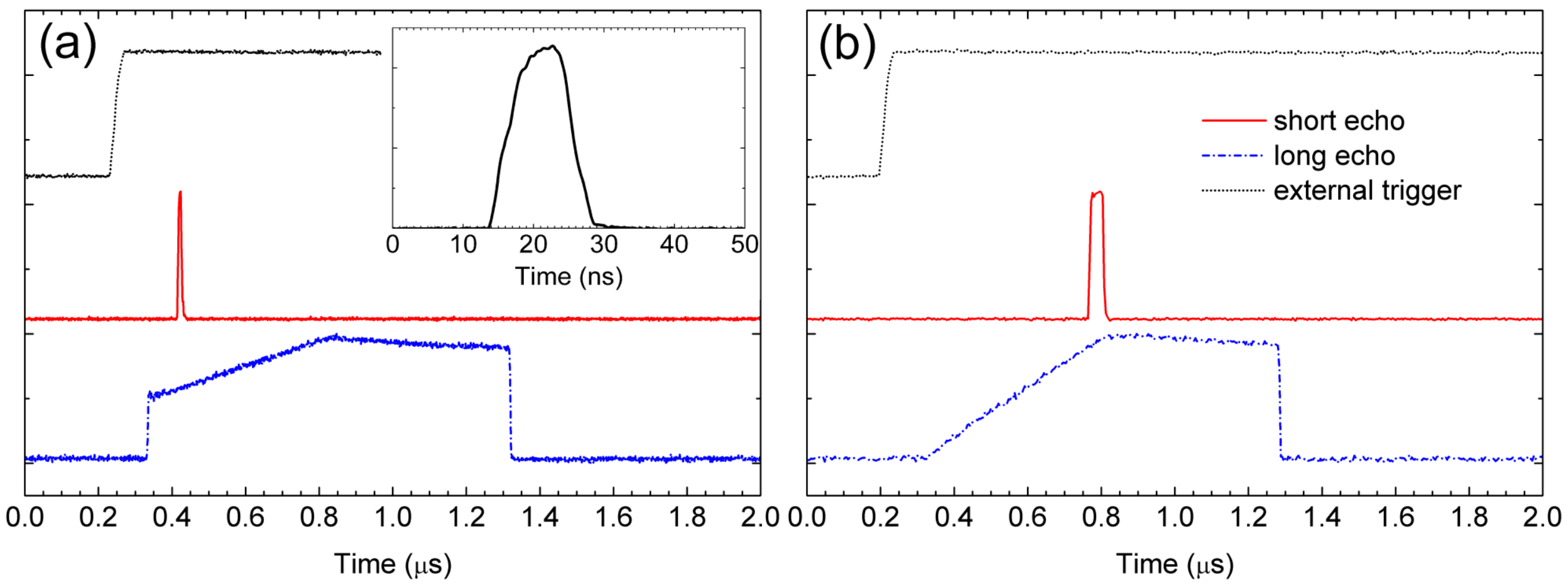




\section{LIDAR Echo Emulator Future Developments}

\section{HOLDON Project}
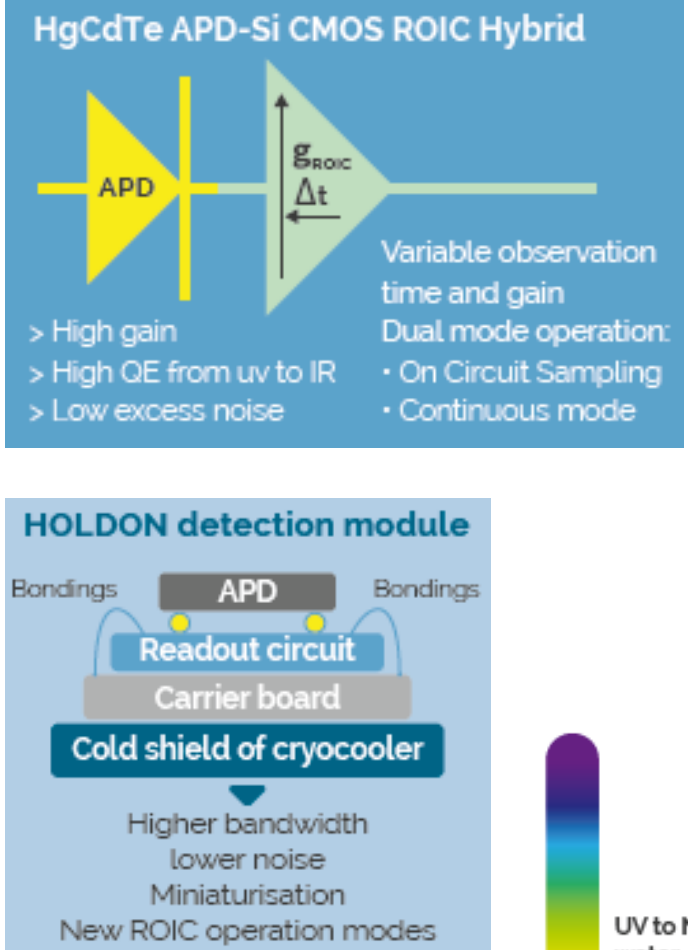
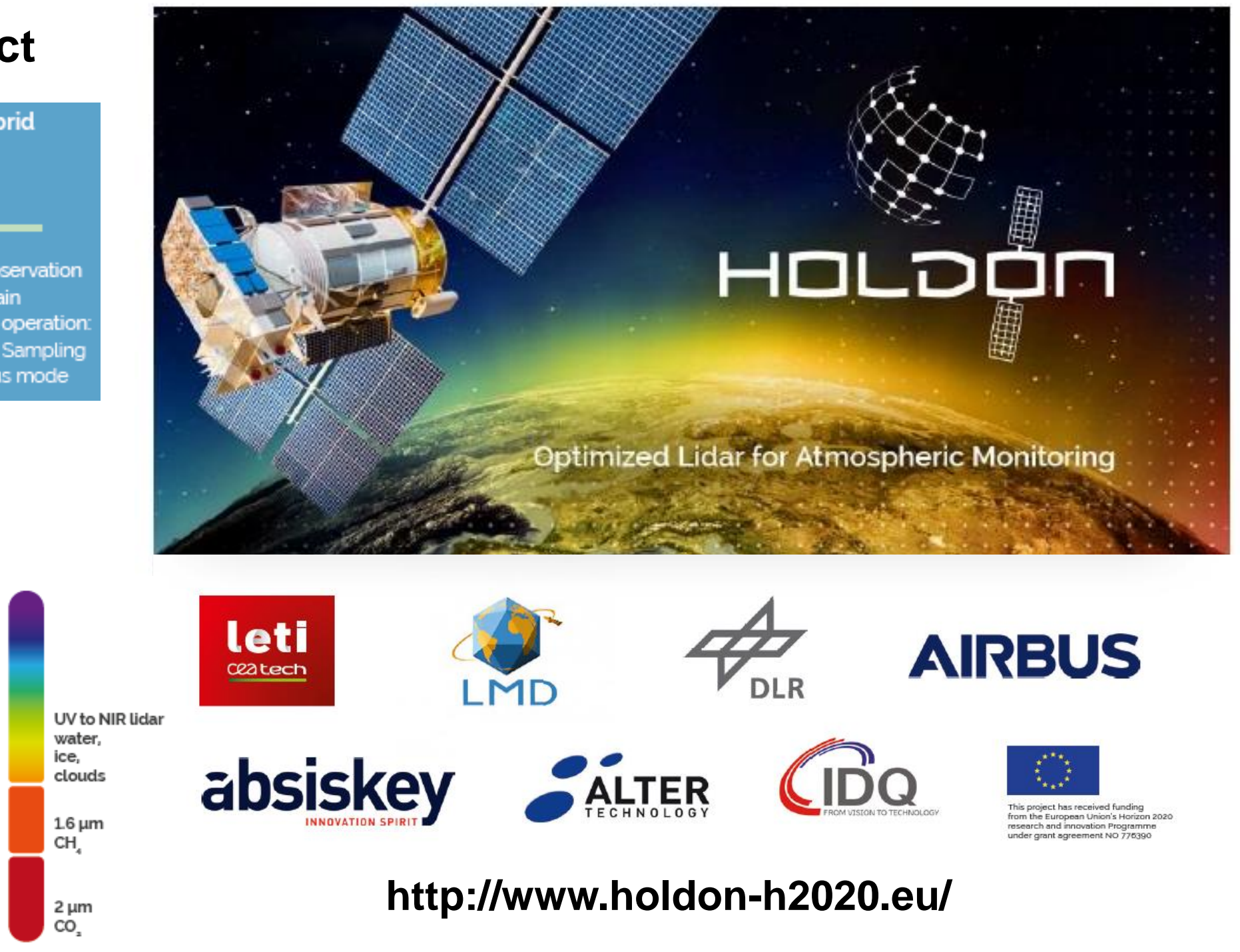

\section{leti}

ceatech

absiskey

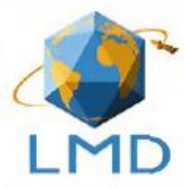

SÁLTER

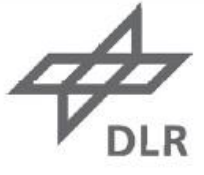

$(100$

\section{AIRBUS}

http://www.holdon-h2020.eu/ 


\section{LIDAR Echo Emulator Future Developments}

$355 \mathrm{~nm}$

$532 \mathrm{~nm}$

$1064 \mathrm{~nm}$

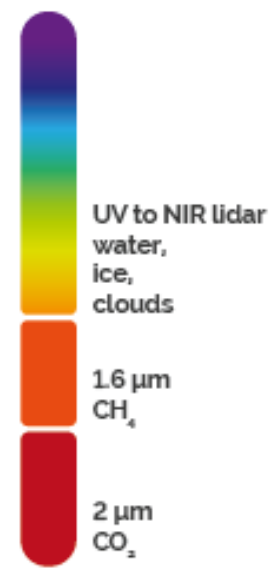

\begin{tabular}{|l|c|}
\multicolumn{1}{|c|}{ Objective 2 } & KPI \\
\hline Wavelength range & $355,532,1064 \mathrm{~nm}$ \\
\hline Long echo duration & $270 \mu \mathrm{s}$ \\
\hline Long echo optical dynamic range & 0.1 to $25 \mathrm{pW}$ \\
\hline Short echo duration & $<10 \mathrm{~ns}$ \\
\hline Short echo optical dynamic range & 0.2 to $200 \mathrm{nW}$ \\
\hline Short echo adjustment step & $<2 \mathrm{~ns}$ \\
\hline Short terms temporal jitter (10 s) & $<+/-2 \mathrm{~ns}$ \\
\hline Long terms temporal jitter (1 hour) & $<+/-10 \mathrm{~ns}$ \\
\hline Short echo falling edge tail (wrt peak) & $<+/-3 \%$ \\
\hline Long terms radiometric stability (1 hour) & $80 \mathrm{~K}$ \\
\hline Nominal operating temperature & $80-180 \mathrm{~K}$ \\
\hline Reachable temperature range & 0 to $20 \mathrm{~V}$ \\
\hline HgCdTe APD adjustable bias range & $5 \mathrm{~ns}$ to $10 \mu \mathrm{s}$ \\
\hline Observation time range in OCS mode & Typically from 1 to 100 samples \\
\hline On chip accumulation range in OCS mode & \\
\hline
\end{tabular}

\section{http://www.holdon-h2020.eu/}




\section{CONCLUSIONS}

- The Lidar echo emulator was designed and developed as a compact and standalone elegant breadboard.

- It delivers two echoes, short and long one to emulate the returns of the lidar system as the output into the Optical fiber (FC/APC).

- Multiple returns could be emulated too.

- It is easy configurable with the delivered software. The parameters of the Lidar echo emulator can be customized.

\begin{tabular}{|c|c|}
\hline Item & Value \\
\hline Peak wavelength & $1064 \mathrm{~nm} \pm 5 \mathrm{~nm}$ \\
\hline \multirow[t]{2}{*}{ Pulse width } & $5 \mathrm{~ns}-1 \mu \mathrm{s}$ (short echo) \\
\hline & $1 \mu \mathrm{s}-1 \mathrm{~ms}$ (long echo) \\
\hline Pulsed output power & $100 \mathrm{fW}-1100 \mathrm{~mW}$ \\
\hline Repetition rate & from $10 \%$ of Duty Cycle to $1 \mathrm{~Hz}$ \\
\hline Short echo falling tail & $50 \mathrm{~dB}$ within $8 \mathrm{~ns}$ \\
\hline $\begin{array}{l}\text { Optical power difference } \\
\text { between both pulses }\end{array}$ & from $0 \mathrm{~dB}$ to $100 \mathrm{~dB}$ \\
\hline
\end{tabular}




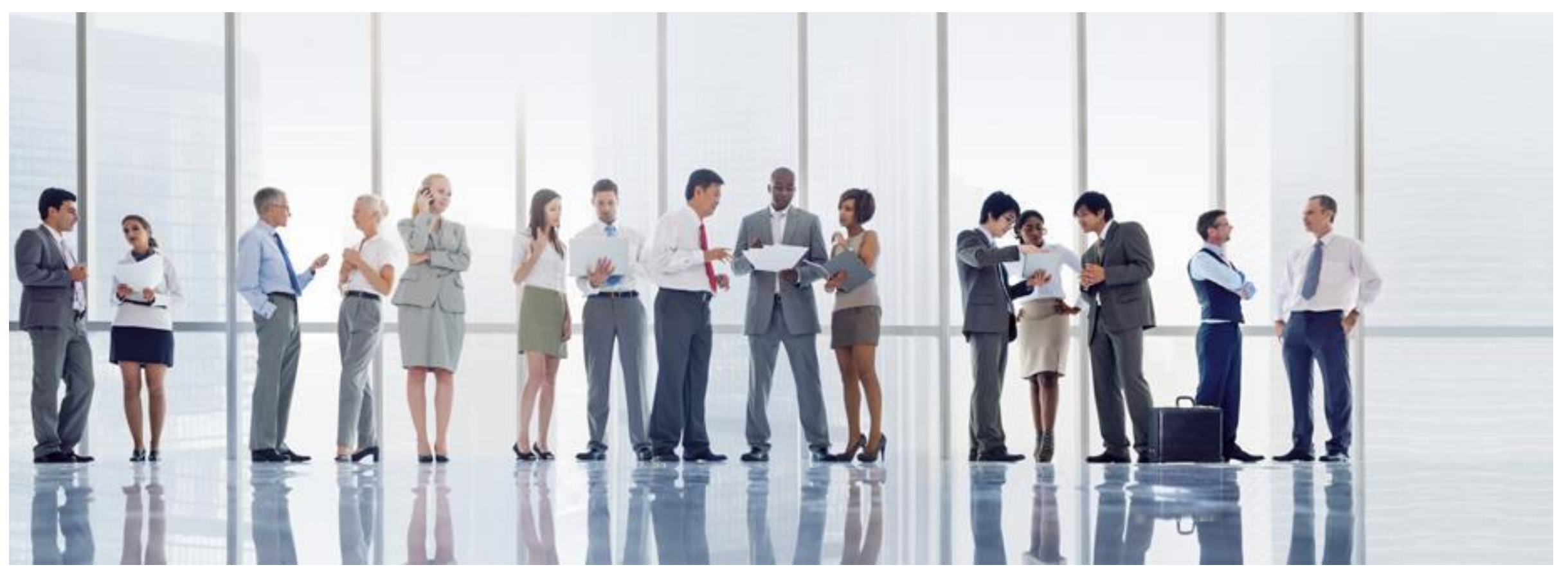

\section{THANK YOU}

